Monatshefte für Chemie 136, 325-345 (2005)

DOI 10.1007/s00706-004-0280-9

\title{
Vibrational Excitons in CH-Stretching Fundamental and Overtone Vibrational Circular Dichroism Spectra ${ }^{\dagger}$
}

\author{
Roberto Gangemi ${ }^{1,2}$, Giovanna Longhi ${ }^{1,2}$, France Lebon ${ }^{1,2}$, \\ Sergio Abbate ${ }^{1,2, *}$, and Leo Laux ${ }^{3}$ \\ 1 Dipartimento di Scienze Biomediche e Biotecnologie, Università di Brescia, \\ 25123 Brescia, Italy \\ 2 INFM-Istituto Nazionale di Fisica della Materia, 25123 Brescia, Italy \\ 3 Lockheed Martin Space Systems Company, Sunnyvale, Corporation, Palo Alto, \\ California, 94089 USA
}

Received September 16, 2004; accepted (revised) November 26, 2004

Published online March 8, 2005 C Springer-Verlag 2005

Summary. A set of vibrational circular dichroism (VCD) spectra in the $\mathrm{CH}$-stretching fundamental region for about twenty compounds belonging to the class of essential oils was empirically analyzed by the use of a sort of vibrational exciton mechanism, involving three centers. Through a general formula applicable to many coupled dipole oscillators, the rotational strengths of the previously identified vibrational excitons are evaluated. The results are then critically reviewed by the use of recent $a b$ initio methodology, as applied to selected molecules of the original set. Further insight is gained by model calculations adding up the contribution of the coupling between electric dipole moments associated with normal mode behavior and that of the polarizability from polarizable groups. The former part is responsible for the excitonic behavior of the VCD spectra. For the same selected molecules we have also investigated whether some excitonic behavior is taking place in the second overtone region, and have concluded that this is not the case.

Keywords. Circular dichroism; Terpenoids; Ab initio calculations; IR spectroscopy.

\section{Introduction}

The exciton concept has been largely used in the context of solid-state physics [1] to designate delocalized excited electronic states, either denoting excited electrons close to the corresponding holes (Frenkel-type or molecular excitons) or independent hole/electron excitations (Mott-Wannier excitons). The Frenkel-

\footnotetext{
* Corresponding author. E-mail: abbate@med.unibs.it

$\dagger$ We dedicate this work to the memory of late Professor Al Moscowitz
} 
type exciton model [2] has been applied by Moffitt [3] in the context of circular dichroism (CD) spectroscopy to first interpret the CD spectra of polypeptides and then by Harada, Nakanishi, and Berova to describe the interaction between two (or more) identical, strongly absorbing chromophores (i.e. electronic states), e.g., two benzoate groups [4]. The two chromophores being chirally disposed, a CD couplet of bands of opposite sign is generated, the characteristics of which (intensity or $A$-value, frequency separation, etc.) can be simply related to the relative distance and orientation of the chromophores, and, as a consequence, to the conformation and/or the configuration of the molecule under study. The implementation and use of the exciton concept has been so widespread and successful, as to generate an "exciton chirality method", which is amongst the most used approaches for analyzing and using CD spectra [4]. In vibrational circular dichroism (VCD) the expression "coupled oscillators" has been introduced early in the recent history of VCD, rather than that of vibrational excitons, to describe situations that are reminiscent of exciton-type behavior [5-7]. Shortly thereafter, in a thorough study of the VCD spectra in the $\mathrm{CH}$-stretching region of terpenes, cyclic ketones, cyclic amines, and alcohols belonging to the class of essential oils, a triplet of signals of alternating sign common to the VCD of all of the molecules studied was identified, describing the interaction of vibrational states residing on three close chemical units, a $\mathrm{CH}_{2}$, another $\mathrm{CH}_{2}$ next to it, and a $\mathrm{C}^{*}-\mathrm{H}$ bond originating from a stereogenic carbon atom next to the two methylene units, and proving some usefulness in conformational analysis [8]. This threestate vibrational exciton system, as presented in Ref. [8], was identified on an empirical basis, but in Ref. [9] a model has been developed to characterize the vibrational excitons for the $\mathrm{CH}_{2} \mathrm{CH}_{2} \mathrm{C}^{*}-\mathrm{H}$ fragment present in all the investigated molecules $[8,9]$ and to compare with current theories for the interpretation of VCD.

Some ten years after that study, VCD data down to the mid-IR deformation and bending region have started to be understood on the basis of $a b$ initio calculations: Bour and Keiderling [10] have studied for some model molecules the conditions under which the full $a b$ initio results could be reproduced by the approximate coupled-oscillator (or vibrational exciton) approach, their conclusion being that the vibrational states needed to be close in frequency and only slightly dynamically coupled. Our work here has a somewhat similar goal to that of Ref. [10]. In addition we wish to report on the essentials of a model elaborated in Ref. [9], that is a perturbative treatment of normal modes analyzed through a matrix formalism, in line with a matrix method first introduced by Schellman [11]. The application of the derived formulae will be made straightforward by a step-by-step explanation. On some selected examples of the group of molecules studied in Refs. [8] and [9], we have performed $a b$ initio calculations and we will investigate whether some qualitative or even quantitative features of the vibrational exciton-model are traceable there. We will then use model calculations, taking into account two sources of rotational strengths, namely on the one hand vibrational exciton coupling and on the other hand localized $\mathrm{CH}$-stretching vibrations interacting with polarizable groups. Finally we will consider the occurrence and manifestation of vibrational excitons in the VCD spectra in the near infrared (NIR) region, where the overtone transitions of $\mathrm{CH}$-stretching modes appear. 


\section{Results and Discussions}

As mentioned in the introduction, in Ref. [8] a set of about 20 molecules showed remarkably similar VCD spectra in the range $2970-2890 \mathrm{~cm}^{-1}$ : all the molecules possess a triplet of units, namely a $\mathrm{CH}_{2} \mathrm{CH}_{2} \mathrm{C}^{*}-\mathrm{H}$ moiety, that has been put in correspondence with a triplet of VCD bands of alternating signs. For the sake of understanding and comparison we redraw from Refs. [8] and [9] three figures with the superimposed VCD spectra of $(3 R)-(+)$-methylcyclohexanone $(\mathbf{1})$ and 2,2,6,6- $\mathrm{d}_{4}-$ $(3 R)-(+)$-methylcyclohexanone (1-d $\left.\mathbf{d}_{\mathbf{4}}\right)$ (Fig. 1), (3R)-(+)-tert-butylcyclohexanone

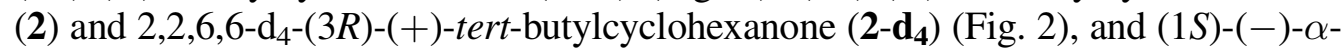
pinene (3) and (1S)-(-)- $\beta$-pinene (4) (Fig. 3), together with their transmittance spectra in the aliphatic $\mathrm{CH}$-stretching fundamental region, between 3000 and $2800 \mathrm{~cm}^{-1}$.

The reason for this choice of data out of the many from Refs. [8] and [9] is that deuterated isotopomers are available for the cyclohexanones, and there is almost no doubt about the conformations of pinenes. In the case of $\mathbf{2}$ we considered the conformation with equatorial tert-butyl substituent. In the case of 1 we considered the two conformations with axial and equatorial $\mathrm{CH}_{3}$, as explained later (see also Ref. [12]). Figures 1 and 2 report on a behavior that is typical of the fragment being part of what we call here "chair A" (Fig. 4A), whereas Fig. 3 corresponds to the fragment belonging to what we call here "chair B" (Fig. 4B), as is clear assuming the most probable conformation for the three types of molecules (see also Refs. [8] and [9]).

The present pattern for chair B was chosen here for a perfect tetrahedral condition; the conformation of $\mathbf{4}$ in the relevant fragment is not so similar to chair Btype (see below); other examples of chair B-type compounds may be found in Refs. [8] and [9]. For chair A, one observes a $(+,-,+)$ pattern for the three most intense VCD bands in order of decreasing wavenumber; for chair B one observes a $(-,+,-)$ pattern. The VCD pattern is relatively large in intensity, with rotational strengths in the order of $10-100 \times 10^{-45} \mathrm{esu}^{2} \mathrm{~cm}^{2}$, and does not seem to be qualita-<smiles>C[C@H]1CCCC(=O)C1</smiles><smiles>CC(C)(C)[C@H]1CCCC(=O)C1</smiles>

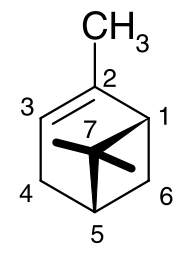

3

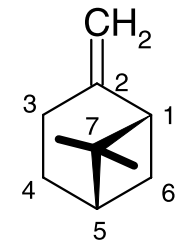

4

Formulae 1 


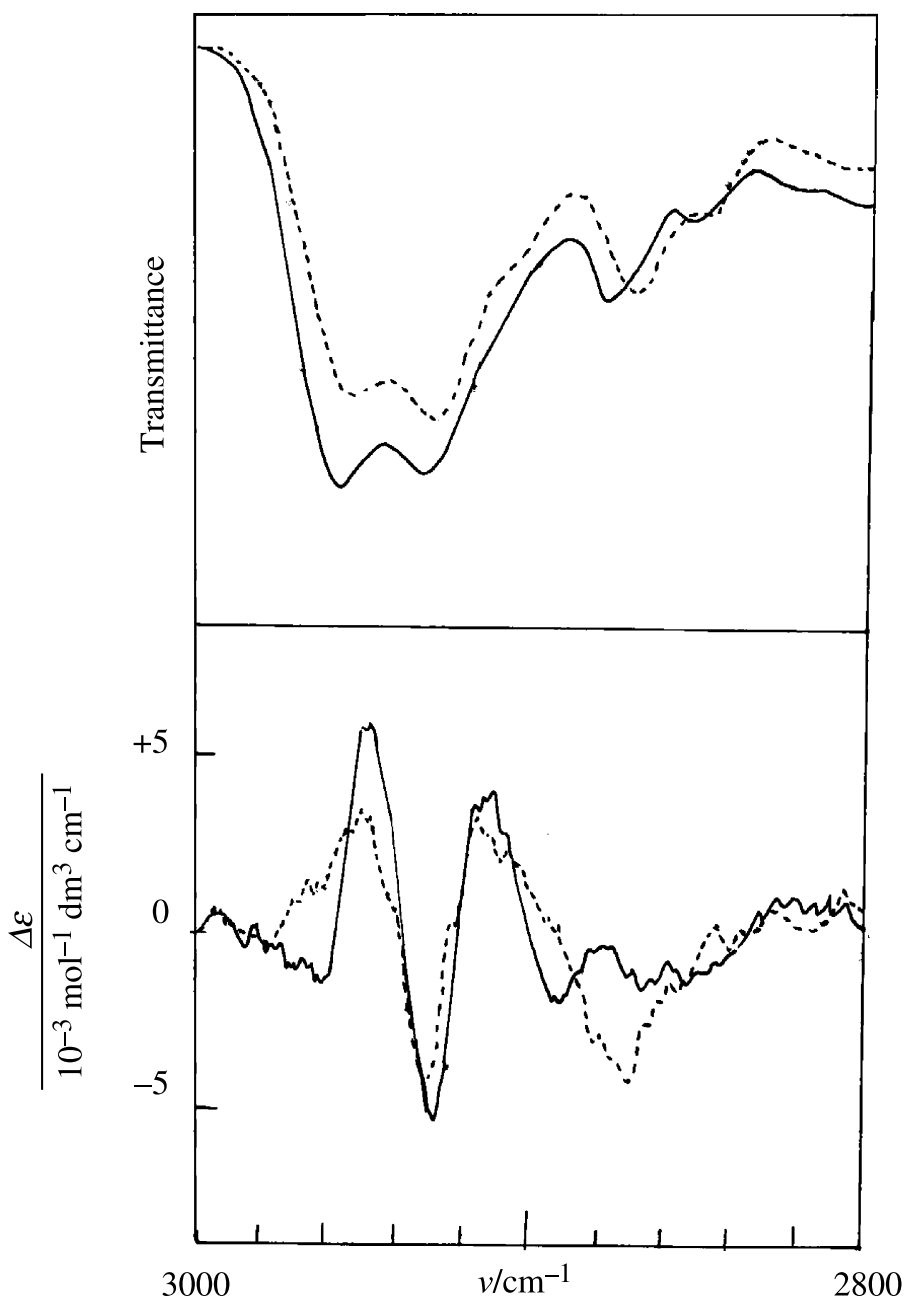

Fig. 1. Experimental transmittance and vibrational circular dichroism spectra in the IR (3000$2800 \mathrm{~cm}^{-1}$ ) for $\mathbf{1}$ (solid lines) and 1-d $\mathbf{d}_{\mathbf{4}}$ (dashed lines); spectra recorded with the instrument of Ref. [9] in $0.060 \mathrm{~mm}$ pathlength $\mathrm{CaF}_{2}$ cell for $0.048 \mathrm{M} / \mathrm{CCl}_{4}$ and $0.045 \mathrm{M} / \mathrm{CCl}_{4}$ solutions

tively affected by changes in the chemical environment of the fragment. Hence, this motivated us [9] to work out a vibrational exciton mechanism, with three excitations residing on the three units, the first one being the antisymmetric $\mathrm{CH}_{2}$-streching of the first unit, the second one the antisymmetric $\mathrm{CH}_{2}$-streching of the next unit, the last one the local $\mathrm{C}^{*}-\mathrm{H}$ stretching, their characteristics being largely unchanged from one molecule to another in the set.

\section{(a) A Model for Vibrational Excitons in the $\mathrm{CH}_{2} \mathrm{CH}_{2} \mathrm{C}^{*}-\mathrm{H}$ Fragment}

Following Ref. [9], we will introduce a model for dealing with all instances where three vibrational excitations in three centers may interact. Let us denote by $\phi_{1}^{0}$ and $\phi_{1}^{1}$ the ground and first vibrational excited state associated with the first centre $\left(\mathrm{CH}_{2}\right.$-antisymmetric stretching), by $\phi_{2}^{0}$ and $\phi_{2}^{1}$ the corresponding states associated 


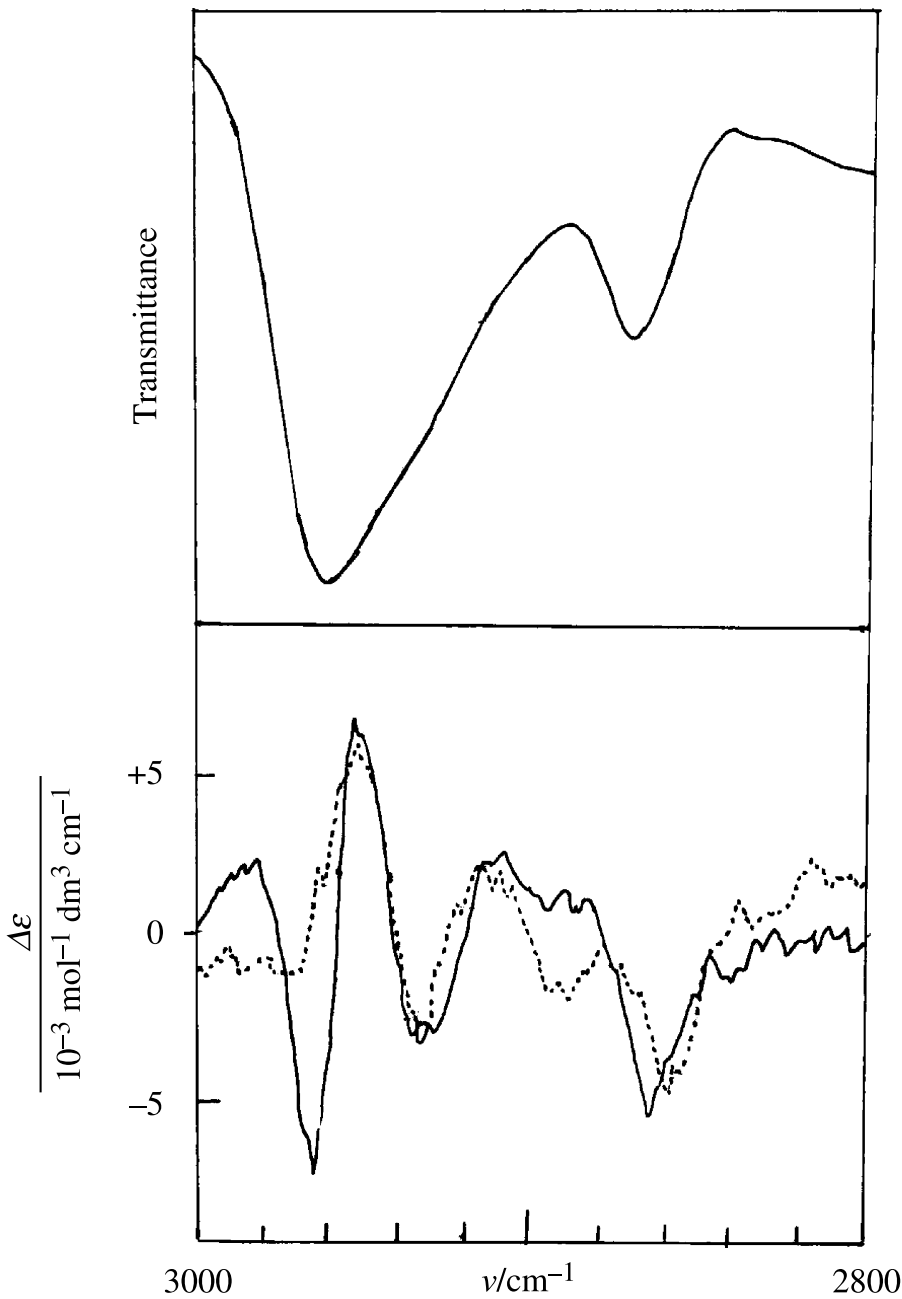

Fig. 2. Experimental transmittance and vibrational circular dichroism spectra in the IR (3000-

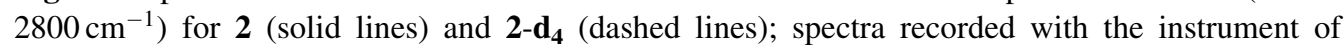
Ref. [9] in $0.060 \mathrm{~mm}$ pathlength $\mathrm{CaF}_{2}$ cell for $0.043 \mathrm{M} / \mathrm{CCl}_{4}$ and $0.045 \mathrm{M} / \mathrm{CCl}_{4}$ solutions

with the second centre $\left(\mathrm{CH}_{2}\right.$-antisymmetric stretching), and by $\phi_{3}^{0}$ and $\phi_{3}^{1}$ the corresponding states associated with the third centre $\left(\mathrm{C}^{*}-\mathrm{H}\right.$-stretching). A first approximation of the three resulting delocalised excitations is given by the direct product $\left|\phi_{j}\right\rangle=\phi_{j}^{1} \phi_{k}^{0} \phi_{l}^{0}(k \neq l \neq j)$ as shown in Eq. (1).

$$
\begin{aligned}
& \left|\phi_{1}\right\rangle=\phi_{1}^{1} \phi_{2}^{0} \phi_{3}^{0} \\
& \left|\phi_{2}\right\rangle=\phi_{1}^{0} \phi_{2}^{1} \phi_{3}^{0} \\
& \left|\phi_{3}\right\rangle=\phi_{1}^{0} \phi_{2}^{0} \phi_{3}^{1}
\end{aligned}
$$

A better approximation of the excitons is given by linear combinations of states given by Eq. (1), namely as shown in Eq. (2).

$$
\left|\Psi_{j}\right\rangle=\frac{1}{\sqrt{N_{j}}} \sum_{k=1}^{3} C_{j k}\left|\phi_{k}\right\rangle
$$




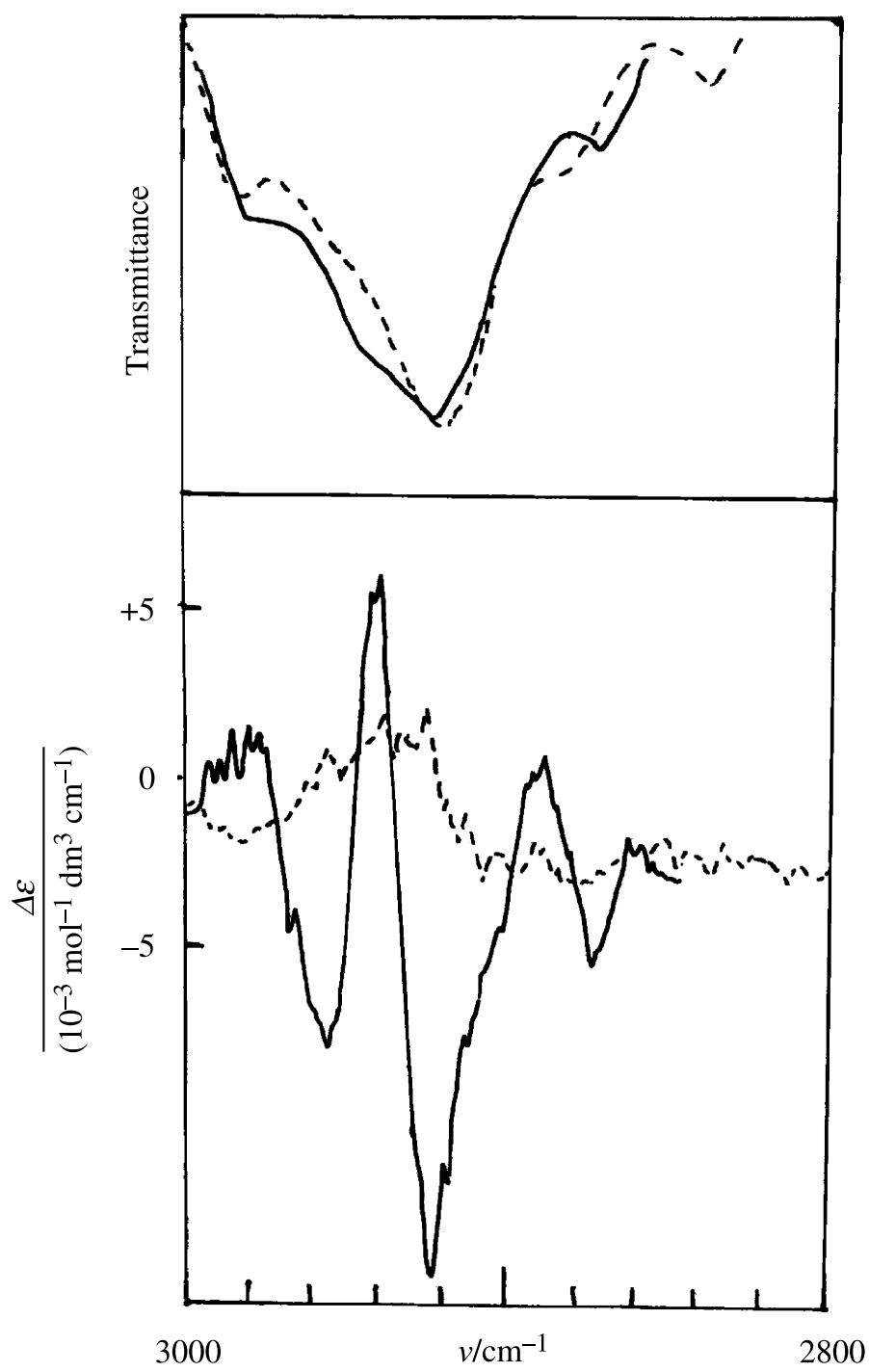

Fig. 3. Experimental transmittance and vibrational circular dichroism spectra in the IR (3000$2800 \mathrm{~cm}^{-1}$ ) for 4 (solid lines) and $\mathbf{3}$ (dashed lines); spectra recorded with the instrument of Ref. [9] in $0.060 \mathrm{~mm}$ pathlength $\mathrm{CaF}_{2}$ cell for $0.049 \mathrm{M} / \mathrm{CCl}_{4}$ in both cases

where

$$
\begin{gathered}
C_{j j}=1 \\
C_{j k}=\left\langle\phi_{j}\left|H^{\prime}\right| \phi_{k}\right\rangle /\left(E_{j}-E_{k}\right)=-C_{k j} \\
N_{j}=\sum_{k=1}^{3} C_{j k}^{2} \\
E_{j}=E_{j}^{0}+\left\langle\phi_{j}\left|H^{\prime}\right| \phi_{j}\right\rangle
\end{gathered}
$$

The values $E_{j}^{0}$ are the energies for the modes of the non-interacting units, and the values $E_{j}$ are for the modes of the interacting units, through the formal inter- 


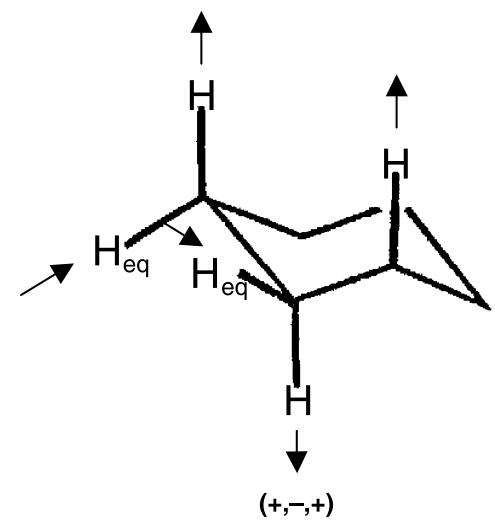

Chair A

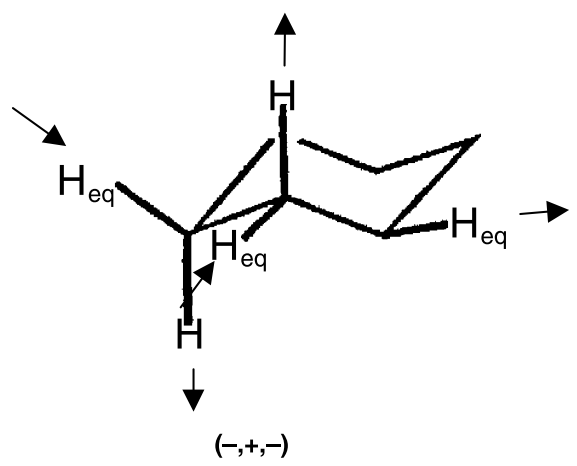

Chair B

Fig. 4. Definition of the geometries associated with chair A-type conformation supposed to correspond to a $(+,-,+)$ triplet of VCD bands and with chair B-type conformation supposed to correspond to a $(-,+,-)$ triplet of VCD bands; superimposed from left to right (see text) is the definition of the three independent vibrational states $\phi_{1}, \phi_{2}$, and $\phi_{3}$

action Hamiltonian $H^{\prime}$. Eqs. $\left(2^{\mathrm{ii}}\right)$ and $\left(2^{\mathrm{iv}}\right)$ are typical of nondegenerate coupled oscillators' theory, as explained in Ref. [9]. In a slightly different notation Eq. (2) may be written as Eq. (3) where $|\Psi\rangle$ and $|\phi\rangle$ denote collectively the set of the three perturbed and unperturbed excitations' wavefunctions respectively; the matrix $\mathbf{T}$, defined as shown in Eq. $\left(3^{\mathrm{i}}\right)$ represents the most general coupling terms $C_{j k}$, under the condition $C_{j j}=1$ and with off-diagonal terms satisfying orthogonality and being such that their product is negligible. We will suppose that Eq. (4) is valid and we define as written in Eq. (5).

$$
\begin{gathered}
|\Psi\rangle=\mathrm{N}^{-1 / 2} \mathbf{T}|\boldsymbol{\phi}\rangle \\
\mathbf{T}=\left[\begin{array}{ccc}
1 & \alpha & \beta \\
-\alpha & 1 & -\gamma \\
-\beta & \gamma & 1
\end{array}\right] \\
E_{1}>E_{2}>E_{3} \\
\left\langle\boldsymbol{\mu}_{j}^{01}\right\rangle=\left\langle\phi_{j}^{0}|\boldsymbol{\mu}| \phi_{j}^{1}\right\rangle
\end{gathered}
$$

It is intuitively acceptable that the same coupling terms $\alpha, \beta$, and $\gamma$ mixing the unperturbed wavefunctions $\left|\phi_{j}\right\rangle$ to generate the perturbed ones $\left|\Psi_{j}\right\rangle$ couple also the transition dipole moments $\left\langle\boldsymbol{\mu}_{j}^{01}\right\rangle$ to generate the rotational strengths $R_{j}$, in the general framework of the coupled-oscillator approach [13]. In Ref. [9] one may find the proof and discussion of the limitations of the ensuing formula for $R_{j}$, which is given in Eq. (6) where c is the speed of light, $E_{j}=\mathrm{h} \nu_{j}$ and $E_{j}^{0}=\mathrm{h} \nu_{j}^{0}$ (h is Planck's constant), $\boldsymbol{r}_{k}(k=1-3)$ define the position of the three units (in what follows they locate the centres of mass of the first $\mathrm{CH}_{2}$, of the second $\mathrm{CH}_{2}$, and of 
$\mathrm{C}^{*}-\mathrm{H}$; minor corrections are obtained by taking them on the three carbon atoms). Further assuming for simplicity that $\beta=0$, that is to say that interactions between $2^{\text {nd }}$ nearest-neighboring units are negligible, one has Eqs. $\left(6^{\mathrm{i}}\right)-\left(6^{\mathrm{iii}}\right)$ which give Eq. (7).

$$
\begin{gathered}
R_{j}=\frac{\pi}{\mathrm{c} \nu_{j} N_{j}} \sum_{k=1}^{3} \sum_{k>l} C_{j k} C_{j l} \nu_{k}^{0} \nu_{l}^{0}\left(\boldsymbol{r}_{k}-\boldsymbol{r}_{l}\right) \cdot\left\langle\boldsymbol{\mu}_{k}^{01}\right\rangle \times\left\langle\boldsymbol{\mu}_{l}^{01}\right\rangle \\
R_{1}=\frac{\pi \nu_{1}^{0} \nu_{2}^{0} \alpha}{\mathrm{c} \nu_{1}\left(1+\alpha^{2}\right)}\left(\boldsymbol{r}_{2}-\boldsymbol{r}_{1}\right) \cdot\left\langle\boldsymbol{\mu}_{2}^{01}\right\rangle \times\left\langle\boldsymbol{\mu}_{1}^{01}\right\rangle \\
R_{2}=\frac{\pi \nu_{2}^{0}}{\mathrm{c} \nu_{2}\left(1+\alpha^{2}+\gamma^{2}\right)} \\
\times\left[-\alpha \nu_{1}^{0}\left(\boldsymbol{r}_{1}-\boldsymbol{r}_{2}\right) \cdot\left\langle\boldsymbol{\mu}_{1}^{01}\right\rangle \times\left\langle\boldsymbol{\mu}_{2}^{01}\right\rangle-\gamma \nu_{3}^{0}\left(\boldsymbol{r}_{3}-\boldsymbol{r}_{2}\right) \cdot\left\langle\boldsymbol{\mu}_{3}^{01}\right\rangle \times\left\langle\boldsymbol{\mu}_{2}^{01}\right\rangle\right] \\
R_{3}=\frac{\pi \nu_{3}^{0} \nu_{2}^{0} \gamma}{\mathrm{c} \nu_{3}\left(1+\gamma^{2}\right)}\left(\boldsymbol{r}_{2}-\boldsymbol{r}_{3}\right) \cdot\left\langle\boldsymbol{\mu}_{2}^{01}\right\rangle \times\left\langle\boldsymbol{\mu}_{3}^{01}\right\rangle \\
R_{2}=-\frac{1}{\nu_{2}}\left(\nu_{1} R_{1}+\nu_{3} R_{3}\right)
\end{gathered}
$$

This is a pleasing result since in all spectra where the relevant pattern of VCD bands is present, $R_{2}$ is opposite to both $R_{1}$ and $R_{3}$, which are observed to have the same sign, and which are associated with the highest and lowest energy transition. In order for one to use Eqs. $\left(6^{\mathrm{i}}\right)-\left(6^{\mathrm{iii}}\right)$, one needs the following information:

i) The values of the Cartesian coordinates defining $\boldsymbol{r}_{j}$. In the following, as in Ref. [9], we adopt the coordinates given in Table 1, which correspond to $\mathrm{CH}$-bond lengths of $1.1 \AA$ and to $\mathrm{C}-\mathrm{C}$-bond lengths of $1.54 \AA$, to tetrahedral interbond angles, and to dihedral angles of $60^{\circ}$ for nearest units (gauche-type relations).

ii) The values for the electric dipole transition moments $\left\langle\boldsymbol{\mu}_{j}^{01}\right\rangle$. We deduced them from an experimental study on local-mode IR and NIR absorption intensities [14], since we believe that Eq. (6) may be generalized to the case of overtone transitions. Limiting ourselves to the fundamental transition, we have, from the data for cyclohexane (Table IV of Ref. [14]), for the local oscillator $\mathrm{C}^{*}-\mathrm{H}$ : $\left|\boldsymbol{\mu}_{3}^{01}\right|=1.33 \times 10^{-2}$ e $\AA=6.38 \times 10^{-20}$ esu cm. This same value may be used for the local $\mathrm{CHs}$ in the two $\mathrm{CH}_{2}$-units. In the latter matrices though the modes to be considered are the $\mathrm{CH}_{2}$-antisymmetric normal modes: so they generate composite dipole moments whose absolute values are $\left|\boldsymbol{\mu}_{1}^{01}\right|=\left|\boldsymbol{\mu}_{2}^{01}\right|=\sqrt{2} \sin (\theta / 2)$ $\left|\boldsymbol{\mu}_{3}^{01}\right|=7.37 \times 10^{-20}$ esu cm $(\theta$ is the tetrahedral angle $)$. The vector $\boldsymbol{\mu}_{3}^{01}$ is then taken to be aligned with $\mathrm{C}^{*}-\mathrm{H}$ from $\mathrm{H}$ to $\mathrm{C}^{*}$, whereas $\boldsymbol{\mu}_{1}^{01}$ and $\boldsymbol{\mu}_{2}^{01}$ were taken to be along directions orthogonal to those bisecting the $\mathrm{HCH}$ angles of the respective $\mathrm{CH}_{2}$ units, from the outward-moving $\mathrm{H}$ towards the inward-moving $\mathrm{H}$ (see Fig. 4).

iii) We need values for $\nu_{1}, \nu_{2}, \nu_{3}$ that are assumed here, as in Ref. [9], to be equal to $\nu_{1}^{0}, \nu_{2}^{0}, \nu_{3}^{0}$. We take them from Table I in Ref. [8]. For chair A we use the experimental values of the frequencies of the observed $(+,-,+)$ triplet in 1-d $\mathbf{d}_{4}$, 
Table 1. The Cartesian coordinates of the $\mathrm{CH}_{2}-\mathrm{CH}_{2}-\mathrm{C}^{*} \mathrm{H}$ fragments in chair $\mathrm{A}$ and $\mathrm{B}$ conformations given in units of $\AA^{\mathrm{a}}$

\begin{tabular}{cccc}
\hline Atom & $x$ & $y$ & $z$ \\
\hline Chair A & & & \\
$\mathrm{C}_{1}$ & 1.2489 & 0.7212 & -0.5100 \\
$\mathrm{C}_{2}$ & 0.0 & 0.0 & 0.0 \\
$\mathrm{C}_{3}$ & -1.2489 & 0.7212 & -0.5100 \\
$\mathrm{H}_{1}$ & 1.2489 & 0.7212 & -1.6200 \\
$\mathrm{H}_{2}$ & 2.1467 & 0.2027 & -0.1433 \\
$\mathrm{H}_{3}$ & 0.0 & 0.0 & 1.1000 \\
$\mathrm{H}_{4}$ & 0.0 & -1.0370 & -0.3667 \\
$\mathrm{H}_{5}$ & -1.2489 & 0.7212 & -1.6200 \\
$\mathrm{Chair}$ & & & \\
$\mathrm{C}_{1}$ & 1.2489 & 0.7212 & 0.5100 \\
$\mathrm{C}_{2}$ & 0.0 & 0.0 & 0.0 \\
$\mathrm{C}_{3}$ & -1.2489 & 0.7212 & 0.5100 \\
$\mathrm{H}_{1}$ & 1.2489 & 0.7212 & 1.6200 \\
$\mathrm{H}_{2}$ & 2.1467 & 0.2027 & 0.1433 \\
$\mathrm{H}_{3}$ & 0.0 & 0.0 & -1.1000 \\
$\mathrm{H}_{4}$ & 0.0 & -1.0370 & 0.3667 \\
$\mathrm{H}_{5}$ & -2.1467 & 0.2027 & 1.1433 \\
\hline
\end{tabular}

${ }^{a} C_{1}, C_{2}, C_{3}$, are defined from left to right in Fig. 4

namely $\varpi_{1}=\nu_{1} / \mathrm{c}=2950 \mathrm{~cm}^{-1}, \quad \varpi_{2}=\nu_{2} / \mathrm{c}=2930 \mathrm{~cm}^{-1}, \quad \varpi_{3}=\nu_{3} / \mathrm{c}=$ $2910 \mathrm{~cm}^{-1}$. For chair B we use the experimental values of the frequencies of the observed $(-,+,-)$ triplet in 4 , namely $\varpi_{1}=\nu_{1} / \mathrm{c}=2956 \mathrm{~cm}^{-1}, \varpi_{2}=\nu_{2} / \mathrm{c}=$ $2939 \mathrm{~cm}^{-1}, \varpi_{3}=\nu_{3} / \mathrm{c}=2922 \mathrm{~cm}^{-1}$.

iv) Last, we need the values for $\alpha$ and $\gamma$ in the interaction matrices $\mathbf{T}$. We take for chair A and B as shown below.

$$
\mathbf{T}=\left[\begin{array}{ccc}
1 & 0.1 & 0 \\
-0.1 & 1 & 0.1 \\
0 & -0.1 & 1
\end{array}\right] \quad \mathbf{T}=\left[\begin{array}{ccc}
1 & 0.1 & 0 \\
-0.1 & 1 & 0.1 \\
0 & -0.1 & 1
\end{array}\right]
$$

The absolute values for $\alpha$ and $\gamma$ are chosen to be an order of magnitude smaller than the diagonal terms; they are not too far from those determined by the solution of the normal mode equations on 1, based on the empirical force-field of Fuhrer et al. [15], as done in Ref. [9]. The signs are those providing the correct sign pattern for the triplets in the two cases.

If we use in Eqs. $\left(6^{\mathrm{i}}\right)-\left(6^{\mathrm{iii}}\right)$ the information given in points $\left.i\right)$ through iv), we obtain for chair A

$$
\begin{array}{lll}
R_{1} \quad R_{2} \quad R_{3} \\
\hline 10^{-45} \mathrm{esu}^{2} \mathrm{~cm}^{2}
\end{array}
$$

$\begin{array}{llll}\text { Calc. } \quad 38.9 & 69.9 & 31.0\end{array}$

$\begin{array}{llll}\text { Exp. } & 43.6 \quad 32.5 \quad 25.3\end{array}$ 
(for 1, as evaluated from the VCD spectra of Refs. [8] and [9]) and for chair B we have

$$
\begin{array}{ll}
R_{1} \quad R_{2} \quad R_{3} \\
\hline 10^{-45} \mathrm{esu}^{2} \mathrm{~cm}^{2}
\end{array}
$$

Calc. $\quad-38.4 \quad 60.3 \quad-21.8$

Exp. $\quad-42.6 \quad 60.9-141.0$

(for 4, Ref. [9]).

Before comparing the results of the present method with ab-initio calculations, we would like to remark that the present formalism was especially developed for dealing with the triplet of signals reported in Ref. [8] which had been discussed previously also in Ref. [16]. However, we notice that Eqs. (6) and (7) are more general than the equations for the more standard case of two coupled oscillators $[5,6]$, in which case one substitutes $\gamma=0$ in Eqs. $\left(6^{\mathrm{i}}\right)-\left(6^{\mathrm{iii}}\right)$, so that only the first two equations of the set are nonzero, and can be generalized to deal with a higher number of coupling factors $\alpha, \beta, \gamma, \delta$, etc., arriving thus at a larger matrix $\mathbf{T}$ in Eq. $\left(3^{\mathrm{i}}\right)$.

We also notice that here $|\alpha|=|\gamma| \ll 1$, whereas in the typical electronic case molecular excitons are such that an almost equal amount of excitation is found in two identical interacting centres [4]. Moreover, in that case there is a precise relation between the frequency separation of the $(+)$ and $(-)$ bands and the difference in CD heights of the two components (frequently referred to as the A-factor [4]). These two peculiarities are related and render the vibrational excitons presented in this work different from the electronic excitons. Indeed, whereas equations like those of the set $\left(6^{\mathrm{i}}\right)-\left(6^{\mathrm{iii}}\right)$ are valid also in the electronic $\mathrm{CD}$ case, the frequency splitting from the large dipole moments $\mu_{1}$ and $\mu_{2}$ associated with the electronic transitions is of the order of $\Delta \varpi=\left(\mu_{1} \mu_{2} / r^{3}\right) /$ hc, $r$ being the distance between the two chromophores, h Planck's constant, and c the speed of light. If we applied this expression to the vibrational case, we would have $\mu_{1}=7 \times$ $10^{-20}$ esu cm, $\mu_{2}=\alpha \times 7 \times 10^{-20}$ esu cm $=0.7 \times 10^{-20}$ esu cm (see points i) and iv) above). Assuming $r=1 \AA$, we would have $\Delta \varpi=2 \mathrm{~cm}^{-1}$. This tells us that, due to the small magnitude of the dipole transition moments associated with $\mathrm{CH}$-stretching vibrations and/or to the small coupling between neighboring units, the band separation of the components of couplets or triplets is not easily related to dipole moment interaction and instead is due to other dynamical factors, like valence force-field interactions or van der Waals forces. A more standard case in this respect of a two-centre vibrational exciton has been pointed out in the beginning of VCD [6] in the $\mathrm{OH}$-stretching region of dimethyl-(+)-tartrate, and has been recently discussed again for the $\mathrm{C}=\mathrm{O}-$ and $\mathrm{OH}$-stretching regions by Buffeteau et al. [17].

\section{(b) Ab initio Calculations and Comparison with the Exciton-Model}

Since the algorithm to calculate VCD rotational strengths was first proposed by Stephens [18], the DFT approach has been applied and different basis sets and functionals have been tested [19], so that by now ab initio calculations of VCD spectra have become a reliable and easy-to-handle tool even for non-experts. 
Another important feature of those calculations is that rotational strengths, dipole strengths, and frequencies are calculated within the same packages in a coherent way, whereby all results have a common root.

As a matter of fact the calculations of VCD spectra of 1 [12] and pinenes [20] by that methodology have already been presented, and have been especially discussed for the mid-IR region, between 1800 and $800 \mathrm{~cm}^{-1}$. We repeated them here for the six molecules, the VCD spectra of which were presented in Figs. 1-3, since their conformations are fairly rigid and defined. We have used the GAUSSIAN03 package [21] with the DFT approach, B3LYP functionals, and 6-31g** basis sets. For $\mathbf{1}$ and 1-d $\mathbf{d}_{\mathbf{4}}$ we have run the calculations for two local minimum energy conformers with the $\mathrm{CH}_{3}$ groups on either the equatorial or axial position. In Fig. 5 we report the results of the thermal average of the two conformations (93\% equatorial and $7 \%$ axial), the relative populations being evaluated at RT with the energy difference between the two conformers as calculated from GAUSSIAN03 itself. The results for $\mathbf{2}$ and $\mathbf{2 - d _ { \mathbf { 4 } }}$ are reported in Fig. 6 and are only for those conformers

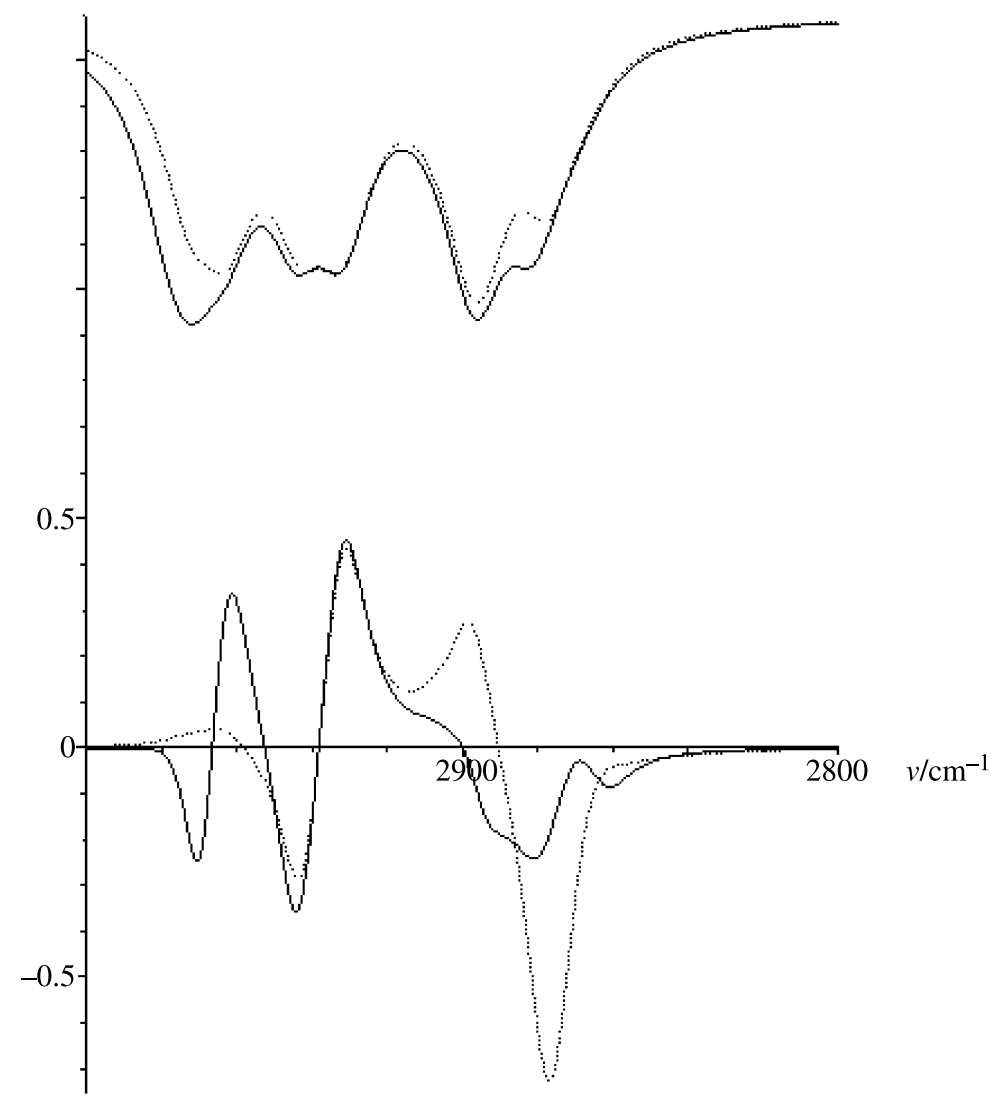

Fig. 5. Calculated transmittance and vibrational circular dichroism spectra in the IR (3000$2800 \mathrm{~cm}^{-1}$ ) for $\mathbf{1}$ (solid lines) and 1-d $\mathbf{d}_{\mathbf{4}}$ (dashed lines); calculations were performed by the use of GAUSSIAN03, as described in the text and in Ref. [22]; units for the ordinate axes are arbitrary; to each calculated line we associated a Lorentzian band shape with band area equal to the calculated rotational strength, centered at the calculated wave number, and with $\gamma=8 \mathrm{~cm}^{-1}$ using a home made computer program 


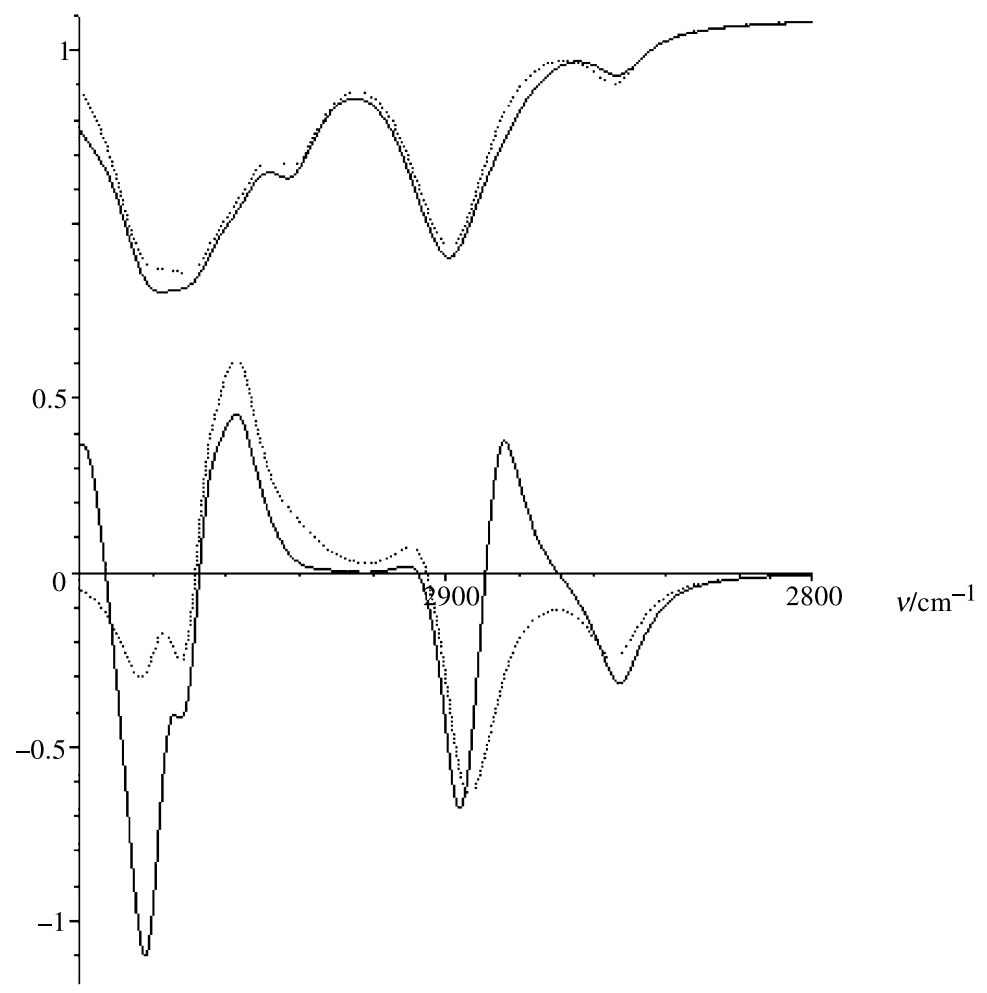

Fig. 6. Calculated transmittance and vibrational circular dichroism spectra in the IR (3000$2800 \mathrm{~cm}^{-1}$ ) for $\mathbf{2}$ (solid lines) and $\mathbf{2}-\mathbf{d}_{\mathbf{4}}$ (dashed lines); calculations were performed by the use of GAUSSIAN03, as described in the text and in Ref. [22]; units for the ordinate axes are arbitrary; to each calculated line we associated a Lorentzian band shape with band area equal to the calculated rotational strength, centered at the calculated wave number, and with $\gamma=8 \mathrm{~cm}^{-1}$, using a home made computer program

in which the tert-butyl substituent is in the equatorial position. Finally in Fig. 7 we give the results for just the lowest-energy conformation of $\mathbf{3}$ and $\mathbf{4}$.

$A b$ initio calculations by most of the available packages do not account for anharmonic effects: as a consequence the calculated frequencies are higher than the experimental ones and need to be corrected. Here we correct them as done in Ref. [22] and thus we subtract $140 \mathrm{~cm}^{-1}$ to account for the effect of an anharmonicity constant $\chi=70 \mathrm{~cm}^{-1}$ at $\Delta v=1$. In Tables 2 and 3 we report both the harmonic and the corrected values under the headings $\omega_{\text {harm }}$ and $\omega_{\text {anharm. }}$. This will also provide a criterion for handling overtone calculations, where anharmonic effects are even more important [22].

Overall and prima facie the calculated VCD spectra compare pretty well with the corresponding experimental ones. However, from comparison of Figs. 1 and 5, one may observe that the highest frequency positive feature of the triplet is predicted to disappear with deuteration. Indeed, if one looks at Table $2^{\mathrm{a}}$, reporting the

${ }^{\text {a }}$ In Table 2 assignments show that the calculated vibrational modes are not only a combination of purely symmetric or antisymmetric $\mathrm{CH}_{2}$-stretchings, but contain extra contributions from either axial or equatorial $\mathrm{CH}$-stretchings. The description of modes is given here according to results of GAUSSIAN03 


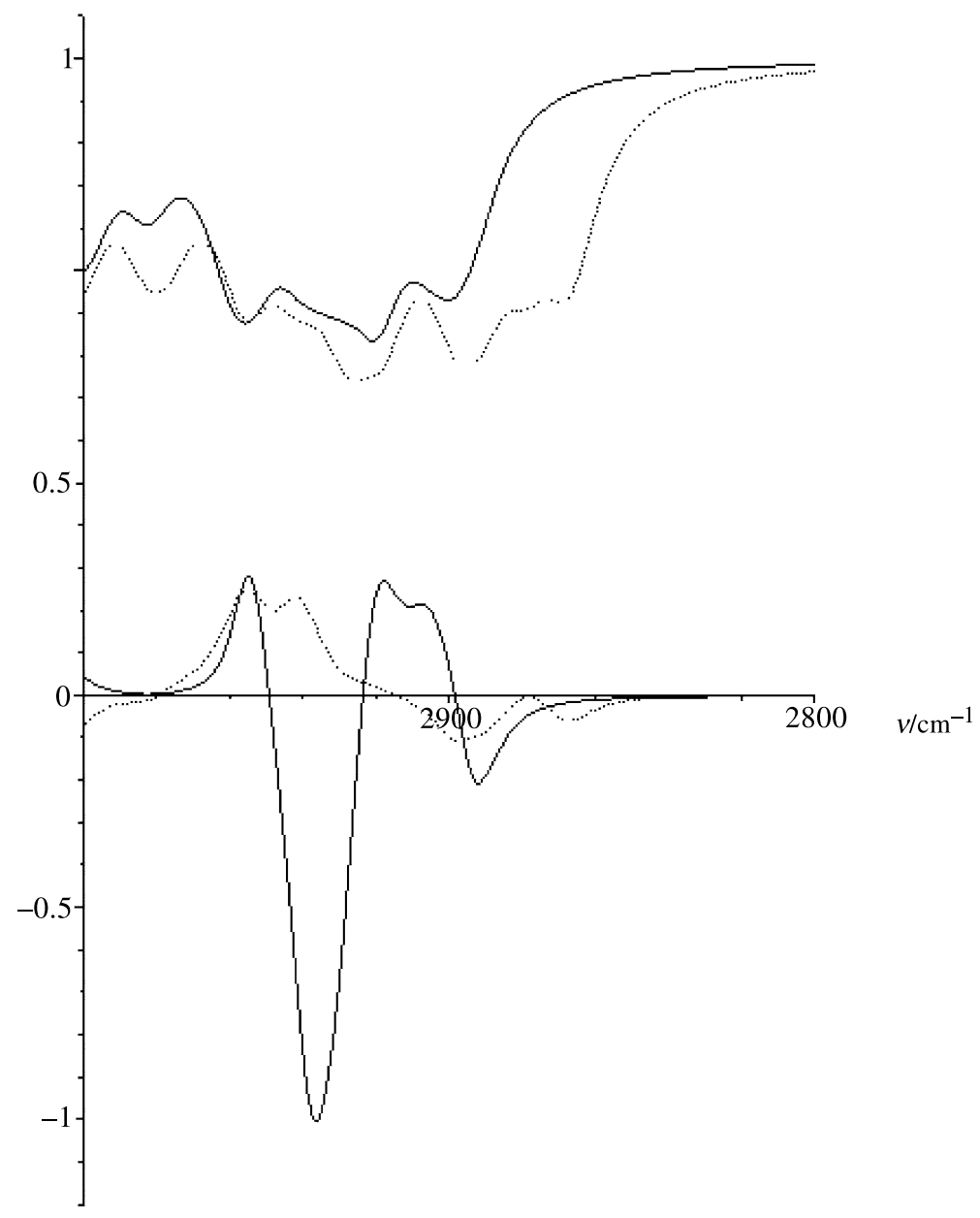

Fig. 7. Calculated transmittance and vibrational circular dichroism spectra in the IR (3000$2800 \mathrm{~cm}^{-1}$ ) for 4 (solid lines) and $\mathbf{3}$ (dashed lines); calculations were performed by the use of GAUSSIAN03, as described in the text and in Ref. [22]; units in the ordinate axes are arbitrary; to each calculated line we associated a Lorentzian band shape with band area equal to the calculated rotational strength, centered at the calculated wave number, and with $\gamma=8 \mathrm{~cm}^{-1}$, using a home made computer program

assignments for the calculated features of the equatorial conformers of the results of Fig. 5, one sees that the feature at $c a .2950 \mathrm{~cm}^{-1}$ for $\mathbf{1}$ is assigned to the $\alpha$-CHs and thus it disappears with deuteration.

Instead, the next two features at $c a 2930 \mathrm{~cm}^{-1}$ and $2910 \mathrm{~cm}^{-1}$ are assigned to the $\mathrm{CH}_{2}$-antisymmetric stretching mode at the $\beta$-position (calculated at $2944 \mathrm{~cm}^{-1}$ ) and to the $\mathrm{CH}_{2}$-antisymmetric stretching mode at the $\gamma$-position that are calculated at 2932 and $2897 \mathrm{~cm}^{-1}$, in interaction, as also assumed in the model of Section (a). According to Table 2 the $\mathrm{C}^{*}-\mathrm{H}$ contribution is found at still lower frequencies mixed together with symmetric $\mathrm{CH}$-stretchings and not with antisymmetric ones; the symmetric $\mathrm{CH}_{2}$ - and $\mathrm{CH}_{3}$-stretching modes have the lowest frequencies. We also note that the symmetric $\mathrm{CH}_{2}$-stretchings have the same local symmetry as the first overtone of the $\mathrm{HCH}$-bending; due to this fact, Fermi resonance effects may 
Table 2. Assignments calculated (GAUSSIAN03) harmonic wavenumbers $\omega_{\text {harm }}\left(\mathrm{cm}^{-1}\right)$, anharmonically corrected wavenumbers $\omega_{\text {anharm }}\left(\mathrm{cm}^{-1}\right)$, dipole strengths $D\left(10^{-40} \mathrm{esu}^{2} \mathrm{~cm}^{2}\right)$, rotational strengths $R\left(10^{-44} \mathrm{esu}^{2} \mathrm{~cm}^{2}\right)$ for $\mathbf{1}$ and $\mathbf{1}-\mathbf{d}_{\mathbf{4}}$ with the methyl in the equatorial position; in the last three columns the results for $D$ and $R$ of model calculations encompassing coupled dipoles and polarizability effects are given and denoted by a star, the last one referring just to polarizability effects (see text)

\begin{tabular}{|c|c|c|c|c|c|c|c|}
\hline Assignments and groups & $\omega_{\text {harm }}$ & $\omega_{\text {anharm }}$ & $D$ & $R$ & $D^{*}$ & $R^{*}$ & $R^{* \mathrm{pol}}$ \\
\hline \multicolumn{8}{|l|}{1} \\
\hline $\mathrm{CH}_{3} \mathrm{symm}+\mathrm{CHax}+\mathrm{C}^{*} \mathrm{H}$ & 3002 & 2862 & 0.97 & -2.80 & 2.48 & -0.44 & 0.15 \\
\hline $\mathrm{CH}_{3} \mathrm{symm}+\mathrm{CHax}+\mathrm{C}^{*} \mathrm{H}$ & 3010 & 2870 & 10.1 & 3.54 & 2.79 & 3.05 & 0.78 \\
\hline antisymm + CHeq & 3019 & 2879 & 18.6 & -5.87 & 16.62 & -6.33 & -1.44 \\
\hline antisymm + CHeq & 3022 & 2882 & 35.4 & -1.12 & 14.45 & -0.93 & -0.26 \\
\hline $\mathrm{C}^{*} \mathrm{H}+\mathrm{CHax}$ & 3035 & 2895 & 33.3 & -11.3 & 17.82 & -5.57 & -0.68 \\
\hline $\mathrm{C}^{*} \mathrm{H}+\mathrm{CHax}$ & 3037 & 2897 & 58.9 & 8.98 & 33.76 & 9.14 & 1.98 \\
\hline antisymm & 3072 & 2932 & 54.6 & 19.3 & 54.30 & 8.21 & 0.43 \\
\hline antisymm & 3084 & 2944 & 49.9 & -18.8 & 61.94 & -9.11 & -0.57 \\
\hline antisymm + CHeq & 3103 & 2963 & 43.2 & 20.6 & 59.88 & 9.52 & -1.15 \\
\hline antisymm + CHeq & 3110 & 2970 & 20.4 & -28.9 & 15.24 & -11.16 & -1.81 \\
\hline $\mathrm{CH}_{3}$ antisymm & 3113 & 2973 & 50.7 & 13.0 & 54.29 & 4.05 & 1.62 \\
\hline $\mathrm{CH}_{3}$ antisymm & 3118 & 2978 & 24.5 & 0.8 & 7.15 & 0.53 & 0.87 \\
\hline \multicolumn{8}{|l|}{ 1-d $\mathbf{d}_{4}$} \\
\hline $\mathrm{CH}^{*}+\operatorname{symm}+\mathrm{CHax}(\gamma)$ & 3006 & 2866 & 4.98 & 3.85 & 3.16 & 3.22 & 0.46 \\
\hline $\mathrm{CH}^{*}-(\mathrm{CH}$ ax $(\gamma)+\mathrm{CH}$ eq $(\beta))$ & 3017 & 2877 & 41.9 & -23.3 & 32.23 & -9.46 & -0.77 \\
\hline $\mathrm{CH}_{3}$ symm & 3035 & 2895 & 37.4 & -3.54 & 19.82 & -1.98 & -0.34 \\
\hline symm + CHax & 3037 & 2897 & 47.0 & 13.4 & 27.18 & 7.92 & 1.29 \\
\hline antisymm $+\mathrm{CHax}$ & 3072 & 2932 & 53.4 & 18.6 & 54.71 & 8.00 & 0.42 \\
\hline antisymm + CHeq & 3083 & 2943 & 45.7 & -15.3 & 58.00 & -8.62 & -0.74 \\
\hline $\mathrm{CH}_{3}$ antisymm & 3103 & 2963 & 53.6 & 1.44 & 70.36 & 3.64 & 0.48 \\
\hline $\mathrm{CH}_{3}$ antisymm & 3112 & 2972 & 36.9 & 0.696 & 54.03 & 1.25 & -0.26 \\
\hline
\end{tabular}

and do highly perturb the symmetric $\mathrm{CH}_{2}$-stretching mode and make the lowest part of the $\mathrm{CH}$-stretching region filled with almost useless information [23].

According to these calculations, there is an exciton couplet associated with the $(-,+)$ couplet calculated for the equatorial conformer of the deuterated isotopomer at 2943 and $2932 \mathrm{~cm}^{-1}$ (corresponding to $\omega_{\text {harm }}=3083$ and $3072 \mathrm{~cm}^{-1}$ in Table 2), which is in correspondence with the experimental $(-,+)$ couplet at 2930 and $2910 \mathrm{~cm}^{-1}$ and is determined by the interaction of $\beta$ - and $\gamma-\mathrm{CH}_{2}$ antisymmetric stretchings. From the quantitative normal mode description in internal coordinates (normalized internal eigenvectors), that we constructed on the basis of the GAUSSIAN03 results, we associate to that couplet the matrix $\mathbf{T}^{\prime}$.

$$
\mathbf{T}^{\prime}=\left[\begin{array}{cc}
1 & -0.20 \\
+0.27 & 1
\end{array}\right] \text { for chair A }
$$

This matrix is deduced from the internal eigenvectors' contributions in the relevant group coordinates of $\mathrm{CH}_{2}$-units $\beta$ and $\gamma$, that we may denote as $\mathrm{d}_{\mathrm{CH}_{2}-\beta}$ and $\mathrm{d}_{\mathrm{CH}_{2}-\gamma}$, as follows: the off-diagonal terms are calculated as the ratios $\mathrm{d}_{\mathrm{CH}_{2}-\gamma} / \mathrm{d}_{\mathrm{CH}_{2}-\beta}$ at 
Table 3. Assignments, calculated (GAUSSIAN03) harmonic wavenumbers $\omega_{\text {harm }}\left(\mathrm{cm}^{-1}\right)$, anharmonically corrected wavenumbers $\omega_{\text {anharm }}\left(\mathrm{cm}^{-1}\right)$, dipole strengths $D\left(10^{-40} \mathrm{esu}^{2} \mathrm{~cm}^{2}\right)$, rotational strengths $R\left(10^{-44} \mathrm{esu}^{2} \mathrm{~cm}^{2}\right)$ for 4 ; in parentheses we give the numbers of the ring carbon atoms following the notation used in the structures at the beginning of the text

\begin{tabular}{|c|c|c|c|c|}
\hline Assignments and groups & $\omega_{\text {harm }}$ & $\omega_{\text {anharm }}$ & $D$ & $R$ \\
\hline $\mathrm{CH}_{2}$ (3) symm & 3033.2 & 2893.2 & 14.79 & -8.32 \\
\hline $\mathrm{CH}_{3} \mathrm{symm} / \mathrm{CH}_{2}$ (3) symm & 3033.6 & 2893.6 & 16.49 & -8.85 \\
\hline $\mathrm{CH}_{3}$ symm & 3039.2 & 2899.2 & 59.13 & 6.67 \\
\hline $\mathrm{CH}_{2}$ (4) symm $-\mathrm{C}^{*} \mathrm{H}(5)$ & 3045.7 & 2905.7 & 33.44 & 9.92 \\
\hline $\mathrm{CH}_{2}$ (4) symm $+\mathrm{C}^{*} \mathrm{H}(5)$ & 3060.2 & 2920.2 & 106.61 & 24.85 \\
\hline$-\mathrm{CH}_{2}$ (6) symm $+\mathrm{C}^{*} \mathrm{H}(1)$ & 3070.8 & 2930.2 & 52.48 & -23.42 \\
\hline $\mathrm{CH}_{2}$ (4) antisymm $+\mathrm{CH}_{2}$ (3) antisymm & 3077.9 & 2937.9 & 21.48 & -48.33 \\
\hline $\mathrm{CH}_{2}$ (6) symm $+\mathrm{C}^{*} \mathrm{H}(1)$ & 3080.6 & 2940.6 & 37.02 & 3.97 \\
\hline $\begin{array}{l}\mathrm{CH}_{3} \text { antisymm } / \mathrm{CH}_{2}(3) \\
\text { antisymm }+\mathrm{CH}_{2} \text { (4) antisymm }\end{array}$ & 3092.4 & 2952.4 & 8.69 & -20.82 \\
\hline $\begin{array}{l}\mathrm{CH}_{3} \text { antisymm } / \mathrm{CH}_{2}(3) \\
\text { antisymm }+\mathrm{CH}_{2} \text { (4) antisymm }\end{array}$ & 3093.7 & 2953.7 & 47.00 & 47.91 \\
\hline $\mathrm{CH}_{3}$ antisymm & 3098.8 & 2958.8 & 68.91 & -6.27 \\
\hline $\mathrm{CH}_{3}$ antisymm, $\mathrm{CH}_{2}(6)$ & 3122.2 & 2982.2 & 40.97 & -0.21 \\
\hline $\mathrm{CH}_{3}$ antisymm, $\mathrm{CH}_{2}$ (6) & 3138.7 & 2988.7 & 18.86 & -1.00 \\
\hline $\mathrm{CH}_{2}$ (6) antisymm & 3140.7 & 3000.7 & 59.79 & 1.72 \\
\hline$=\mathrm{CH}_{2} \mathrm{symm}$ & 3145.4 & 3005.4 & 13.24 & 2.22 \\
\hline$=\mathrm{CH}_{2}$ antisymm & 3227.0 & 3087.0 & 21.57 & 0.17 \\
\hline
\end{tabular}

$2943 \mathrm{~cm}^{-1}$ (term $(1,2)$ ) and $\mathrm{d}_{\mathrm{CH}_{2}-\beta} / \mathrm{d}_{\mathrm{CH}_{2}-\gamma}$ at $2932 \mathrm{~cm}^{-1}$ (term $(2,1)$ ), the $\mathrm{d}_{\mathrm{CH}_{2} \text {-unit }}$ (unit $=\beta$ or $\gamma$ ) being defined as $\mathrm{d}_{\mathrm{CH}_{2} \text {-unit }}^{2}=\left(\mathrm{d}_{\mathrm{CHax}}^{2}+\mathrm{d}_{\mathrm{CHeq}}^{2}\right.$ ). As noticed above $\left|\mathrm{d}_{\mathrm{CHax}}\right|$ and $\left|\mathrm{d}_{\mathrm{CHeq}}\right|$ are found not to be equal, as it was assumed in the empirical approach. One may notice that the off-diagonal terms of this matrix $\mathbf{T}^{\prime}$ are opposite to the $(1,2)$ and $(2,1)$ off-diagonal terms of the $\mathbf{T}$-empirical matrix; this indeed justifies the $\beta-\gamma$ interaction providing a $(-,+)$ couplet (to be associated with the lowest frequency part of the observed $(+,-,+)$ triplet) rather than a $(+,-)$ couplet (to be associated with the higher frequency part of the triplet $(+,-,+)$ ). The same type of considerations can be made for $\mathbf{2}$ and $\mathbf{2}-\mathbf{d}_{\mathbf{4}}$.

The availability of a good description of $\mathrm{CH}$-stretching normal modes from $a b$ initio calculations, i.e. the availability of a full set of coefficients $C_{j k}$, allows one to apply the vibrational exciton model in the form of Eq. (6) to the whole set of $\mathrm{CH}$ oscillators. Still further insight into the origin of rotational strengths may be gathered from a model calculation of the type presented in Ref. [22], following ideas expounded in Ref. [23]. Therein the rotational strength associated with a fundamental vibrational transition was recognized as due to two sources, the oriented interactions of bond dipoles and the oriented interaction of bond dipoles with induced dipoles on a polarizable group. In $\mathbf{1}$ this is the $\mathrm{C}=\mathrm{O}$ bond, particularly because of the lone pairs of the oxygen atom. To the $\mathrm{C}=\mathrm{O}$ bond we associate a cylindrical polarizability tensor $((\alpha))$, the longitudinal term, along the principal reference axis is $2.3 \AA^{3}$, and the transverse terms are $2.0 \AA^{3}$ [22], considering the work of Applequist et al. [24]. $R_{j}$ is then given by Eq. (8) with $\left\langle\boldsymbol{\mu}_{p}\right\rangle$ being defined 
by Eq. $\left(8^{\prime}\right)$ where $\boldsymbol{E}\left(\left\langle\boldsymbol{\mu}_{k}^{01}\right\rangle\right)$ is the electric field associated with the bond transitions dipole moment $\left\langle\boldsymbol{\mu}_{k}^{01}\right\rangle$, the index $k$ running on the $\mathrm{CH}$ bonds. $L_{k j}$ is the entry of the normalized eigenvectors that defines the fractional participation of $k$-th bond stretching to the $j$-th normal mode.

$$
\begin{gathered}
R_{j}=\frac{\pi \nu_{j}}{\mathrm{c}} \sum_{k} \sum_{l>k} L_{k j} L_{l j}\left(\boldsymbol{r}_{k}-\boldsymbol{r}_{l}\right) \cdot\left\langle\boldsymbol{\mu}_{k}^{01}\right\rangle \times\left\langle\boldsymbol{\mu}_{l}^{01}\right\rangle+\frac{1}{2} \sum_{k} L_{k j}\left(\boldsymbol{r}_{p}-\boldsymbol{r}_{k}\right) \cdot\left\langle\boldsymbol{\mu}_{p}\right\rangle \times\left\langle\boldsymbol{\mu}_{k}^{01}\right\rangle \\
\left\langle\boldsymbol{\mu}_{p}\right\rangle=\underline{\underline{\alpha}} \cdot \boldsymbol{E}\left(\left\langle\boldsymbol{\mu}_{k}^{01}\right\rangle\right)
\end{gathered}
$$

The first part of Eq. (8) is similar to Eq. (6), if one substitutes coefficients $C$ with coefficients $L$ and assumes that $\nu_{j} \approx \nu_{k} \approx \nu_{l}$. This means that if one has large couplings, i.e. $L_{k j} \neq 0$ for more than one $k$ (normal modes), the coupling between dipoles is the dominant effect; if just one $L_{k j} \neq 0$ (local modes), the polarizability term (second summation of Eq. (8)) is the dominant one. For 1 and for fundamental vibrational transitions it will be shown below that the coupling between dipoles (i.e. the exciton mechanism) is more important than the local effect; see Ref. [22] for opposite situations, in fundamental and overtone transitions.

If we take for the dipole transition moments of $\beta-, \gamma-$, and methyl- $\mathrm{CH}$ bonds the value of $1.33 \times 10^{-2} \mathrm{e} \AA$ and for $\alpha$-CH bonds the value $0.496 \times 10^{-2}$ e $\AA$ (see Refs. [22] and [14]), we obtain the results of Table 2 and Fig. 8 for $\mathbf{1}$ and 1-d $\mathbf{4}$.

The coefficients $L_{k j}$ are evaluated from the ab initio calculations as well as the values $\nu$, that are corrected for anharmonicity as done above. In Table 2 we also report the value for the rotational strengths $R^{* \text { pol }}$ due to the second term of Eq. (8) and we may see that the major contribution is from interaction of transition bond dipoles and not from polarizability terms, making the assumptions of Refs. [8] and [9] and of the beginning part of this work acceptable. In general one may see that the results of the model are good; this had been observed already in Ref. [22] for $(3 R)-(+)$-methyl-cyclopentanone. The difference between $a b$ initio and model results are due to different evaluation of $\left\langle\boldsymbol{\mu}_{k}^{01}\right\rangle$ : the latter values in the model calculation are from experiment [14], whereas in the ab initio based approach presented above, they are calculated within the package. In fact using the vibrational exciton model on the whole set of $\mathrm{CH}$-bond stretchings, the $(+,-,+)$ triplet observed in $\mathbf{1}$ is obtained; however, the highest frequency band is assigned to methyl CH-stretchings. Why this band is not reproduced by complete DFT ab initio calculations is not clear.

Considering now the case of pinenes, the ab initio prediction of Fig. 7 is good, the calculated VCD spectrum of the $\alpha$-species being calculated with very low intensities compared to that of the $\beta$-species. For the latter, for which we also have good results, instead of $(-,+,-)$ at 2954, 2939, and $2922 \mathrm{~cm}^{-1}$, GAUSSIAN 03 calculations predict a $(+,-)$ couplet at $\omega_{\text {anharm }}=2954$ and $2938 \mathrm{~cm}^{-1}$ (corresponding to $\omega_{\text {harm }}=3093.7$ and $3077.9 \mathrm{~cm}^{-1}$ in Table 3), the latter feature being so intense as to be associated with the $2922 \mathrm{~cm}^{-1}$ negative experimental feature (Fig. 7 and Table 3).

From Table 3 one may appreciate that this couplet originated from a $\mathrm{CH}_{2} / \mathrm{CH}_{2}$ antisymmetric coupling. The $(2 \times 2)$ interaction matrix that may be set up has the same structure as that for $\mathbf{T}^{\prime}$ introduced above; the signs for the calculated VCD 


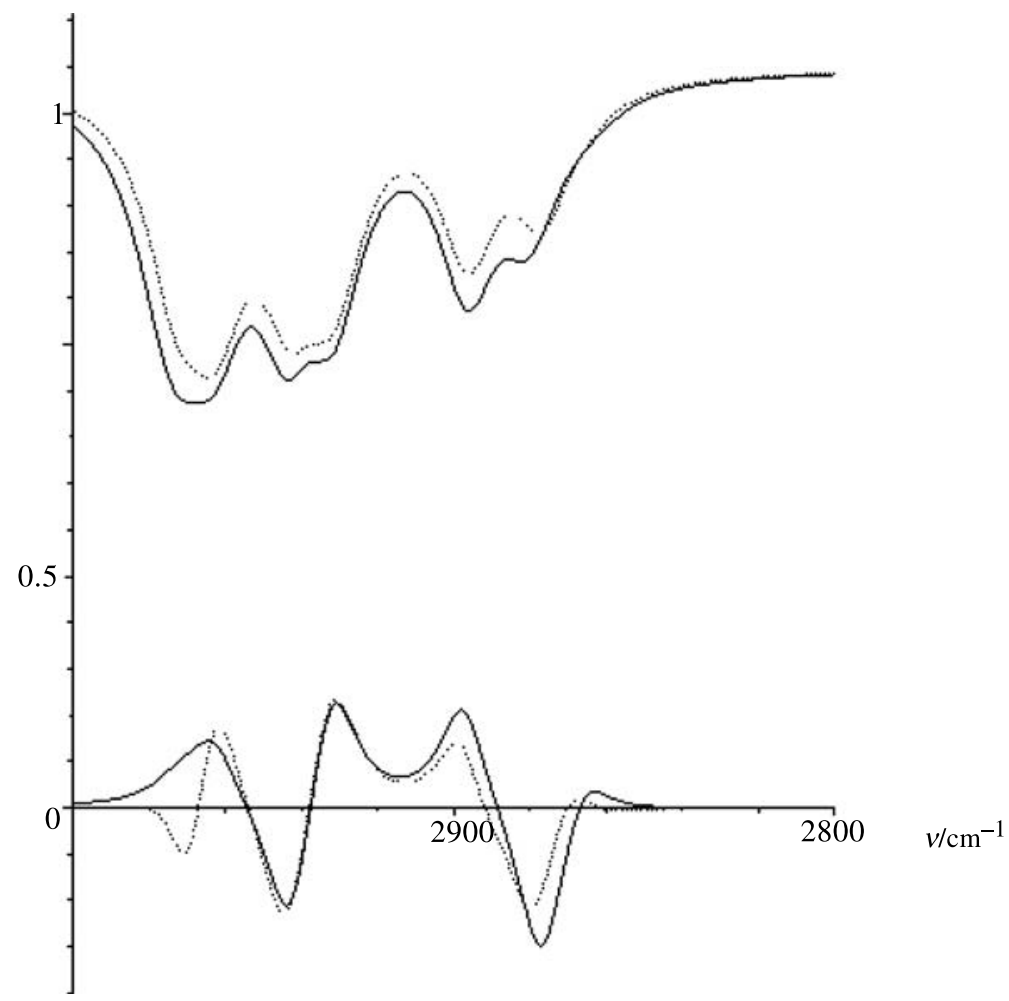

Fig. 8. Calculated transmittance and vibrational circular dichroism spectra in the IR (3000$2800 \mathrm{~cm}^{-1}$ ) for $\mathbf{1}$ (solid lines) and 1-d $\mathbf{4}$ (dashed lines); calculations of frequencies were done as in Figs. 4-7; calculation of dipole and rotational strengths were based on the model describing interacting $\mathrm{CH}$ bond dipoles and polarizability effects as described in the text; units in the ordinate axes are arbitrary; to each calculated line we associated a Lorentzian band shape with band area equal to the calculated rotational strength, centered at the calculated wave number, and with $\gamma=8 \mathrm{~cm}^{-1}$, using a home made computer program

spectrum that are produced for the couplet are opposite, since the conformation of 4 is approximately opposite to that of $\mathbf{1}$ in the $\mathrm{CH}_{2} \mathrm{CH}_{2} \mathrm{C}^{*}-\mathrm{H}$ fragment of relevance. However, we need to say that the predicted conformation for the six-membered ring in $\mathbf{4}$ is quite different from that of $\mathbf{1}$. In the latter case we have a conformation that is in good agreement with that assumed for the $\mathrm{CH}_{2} \mathrm{CH}_{2} \mathrm{C}^{*}-\mathrm{H}$ fragment in Fig. 4 and Table 1 for chair A, whereas in the former case $a b$ initio results are noticeably different, albeit not in contradiction, from those of Table 1 and Fig. 4 for chair B. In the conformation determined via ab initio calculations, the $\mathrm{CH}_{2} / \mathrm{CH}_{2}$ units are predicted with a relative dihedral angle of $26^{\circ}$ instead of $60^{\circ}$ as of Fig. 4.

At this point let us say that GAUSSIAN03 calculations do not support completely the empirical conclusions reached some time ago [8, 9]. However, they still predict that $\mathrm{CH}_{2}$-antisymmetric normal modes of neighboring units can give rise to vibrational excitons, in couplets rather than in triplets. Once more, as for $\mathbf{1}$, the highest frequency band of the triplet of $\mathbf{4}$ is not explained by this ab initio calculation. 
(c) Do Vibrational Excitons Manifest Themselves in NIR-VCD Spectra?

The $\mathrm{CH}$-stretching overtone region for $\Delta v>2$ is thought to be the region of the manifestation of local modes [25, 26], and in this scheme one should not expect, at $\Delta v=3, e . g$., any vibrational exciton there. Recent dynamical studies [27] point out the conditions for the existence and VCD appearance of coupled modes; these studies indicate somewhat exceptional circumstances for the manifestation of coupled modes. However, also the measurement of VCD spectra in the overtone region is rather difficult, due to the small values of the rotational strengths of the VCD bands giving rise to $\Delta \varepsilon$-values of the order of $10^{-3} \mathrm{~mol}^{-1} \mathrm{dm}^{3} \mathrm{~cm}^{-1}$ (even though the $g$-values are of the same order of magnitude as at $\Delta v=1$ ) [27-29].

In Ref. [22] we could arrive at a first interpretation of the VCD spectrum for $\Delta v=3$ of 1 by transferring to $\Delta v=3$ the results from the DFT/B3LYP ab initio calculations at $\Delta v=1$ for the twelve deuterated isotopomers of $\mathbf{1}$, where just one $\mathrm{CH}$ is left undeuterated and the remaining eleven are $\mathrm{CD}$ bonds. The acceptable correspondence between experiment and model is in line with the local mode
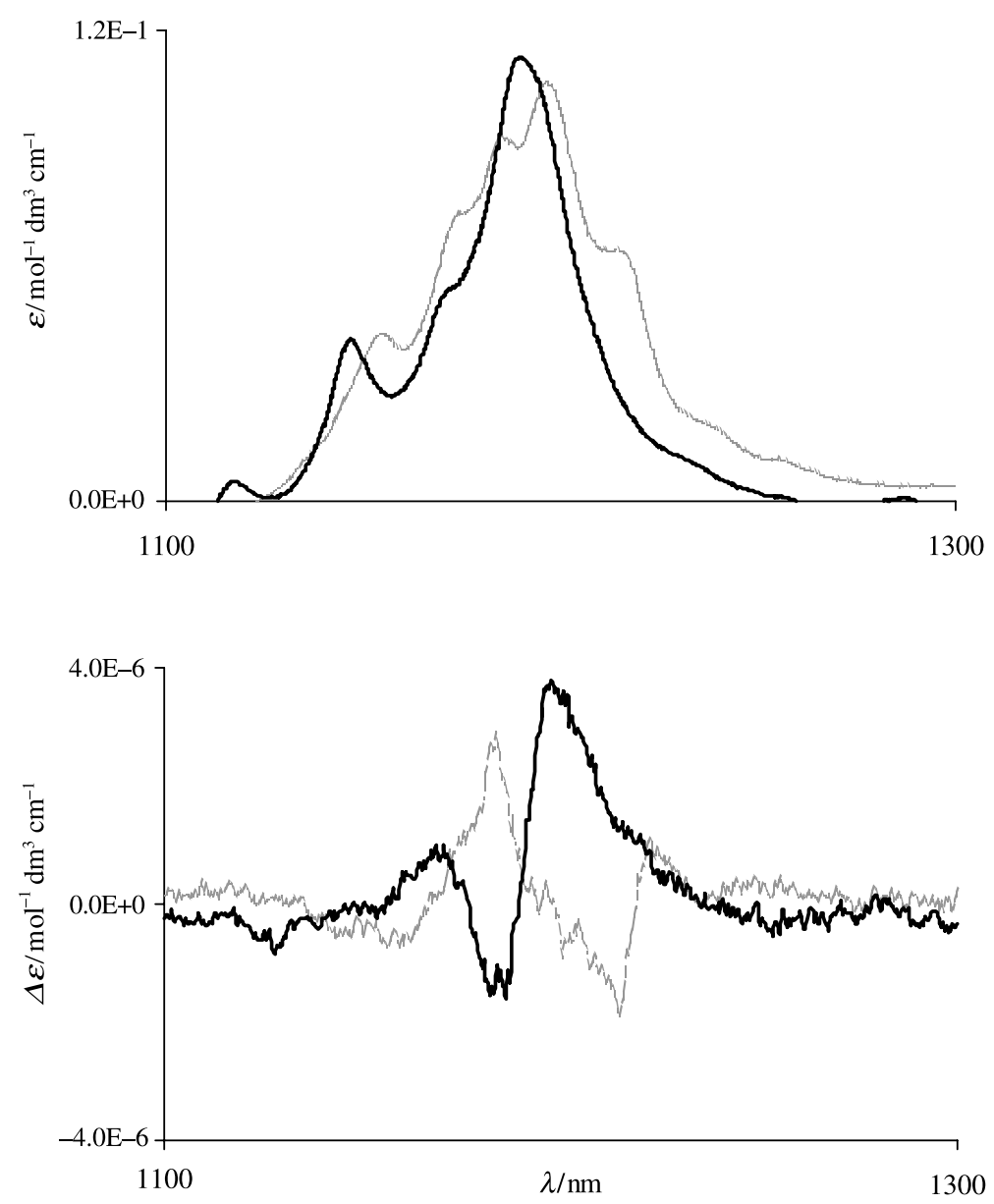

Fig. 9. Experimental transmittance and vibrational circular dichroism spectra in the NIR (1300$1100 \mathrm{~nm}$ ) for 4 (solid lines) and $\mathbf{3}$ (dashed lines); spectra recorded by means of the instrument of Ref. [30] in $0.5 \mathrm{~cm}$ pathlength $\mathrm{SiO}_{2}$ cell for neat liquids in both cases (compare with Ref. [30]) 
hypothesis. Indeed the $\mathrm{CH}$-stretching vibrations of the twelve isotopomers represent the twelve possible local modes for $\mathbf{1}$ and the calculated VCD spectra thereof reflect the vibrational optical activity of isolated $\mathrm{CHs}$ in a chiral environment. In this situation the possible presence of vibrational excitons can be neglected.

A similar correspondence between experiment and model calculations is found for 3 and 4. In Fig. 9 we superimpose the experimental VCD and absorption spectra of the two molecules in the region between 1300 and $1100 \mathrm{~nm}(\Delta v=3)$. The data are taken from Ref. [30] and the VCD spectra are plotted as (1/2) times the difference of the VCD spectra of the $(1 S)$-enantiomers and the VCD spectra of the $(1 R)$-enantiomers, as recently reported also by Nafie et al. [31]. Figure 10 displays the calculations for $\mathbf{3}$ and $\mathbf{4}$, obtained with the same method as in Ref. [22].

The correspondence is acceptable at this almost preliminary point; notice that the calculated VCD spectra of $\mathbf{3}$ and $\mathbf{4}$ look almost enantiomeric, like the experimental ones, and the structuring of the absorption spectra is fairly well reproduced. The assignment of the VCD features based on calculations for $\mathbf{3}$ is: the negative
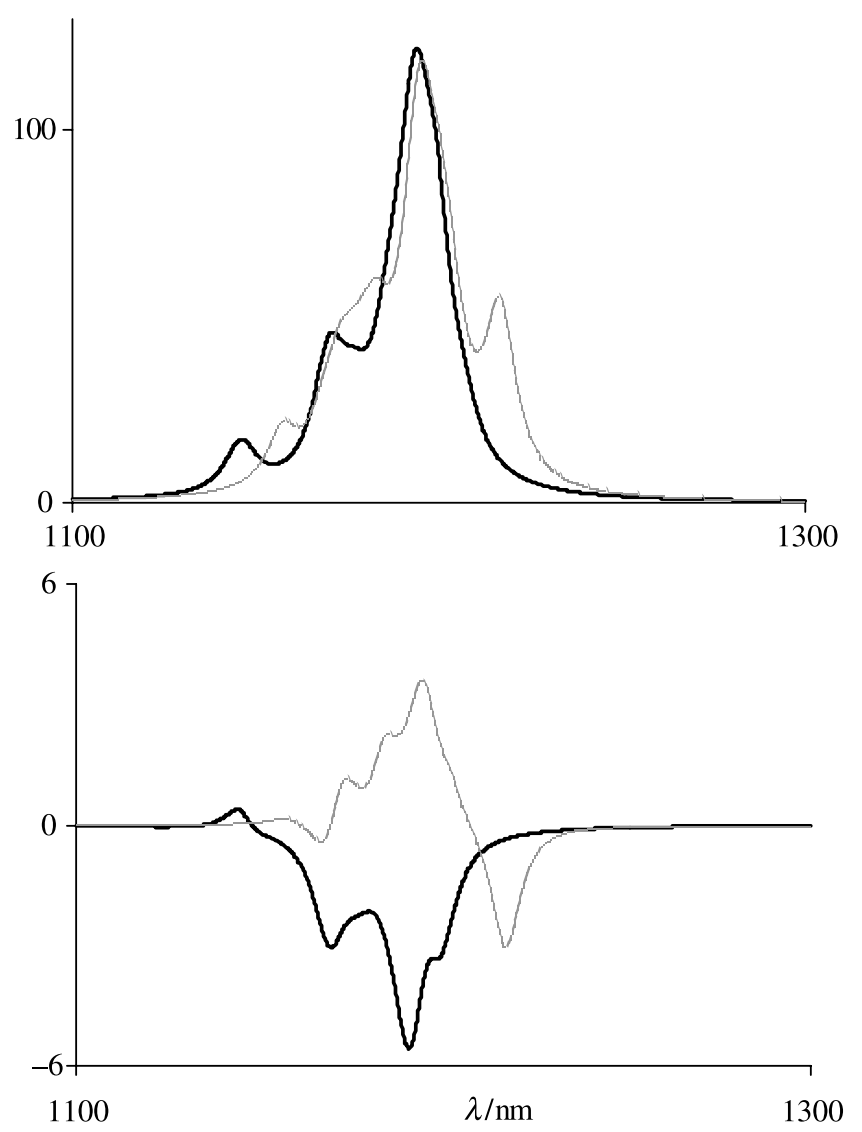

Fig. 10. Calculated transmittance and vibrational circular dichroism spectra in the NIR (1300$1100 \mathrm{~nm}$ ) for 4 (solid lines) and $\mathbf{3}$ (dashed lines); calculations were performed by the use of GAUSSIAN03, as described in the text and in Ref. [22]; units in the ordinate axes are arbitrary; to each calculated line we associated a Lorentzian band shape with band area equal to the calculated rotational strength, centered at the calculated wave number, and with $\gamma=12 \mathrm{~nm}$, using a home made computer program 
VCD band at $c a .1220 \mathrm{~nm}$ corresponds to the local mode at centre 4 , the positive broad structured band between 1210 and $1180 \mathrm{~nm}$ corresponds to $\mathrm{C}^{*}(1) \mathrm{H}, \mathrm{C}^{*}(5) \mathrm{H}$, methyl $\mathrm{CHs}$, and $\mathrm{C}(6) \mathrm{H}$ local modes, the weak negative band at $1175 \mathrm{~nm}$ corresponds to some bridge $\mathrm{CH}_{3}$ local modes. In 4 the calculated order of local modes from high wavelength to low wavelength is: $\mathrm{C}(3) \mathrm{Hs}, \mathrm{C}(4) \mathrm{Hs}, \mathrm{C}^{*}(1) \mathrm{H}, \mathrm{CH}_{3}$, and $\mathrm{C}^{*}(5) \mathrm{H}$. All their rotational strengths are calculated to be negative except a couple of local methyl $\mathrm{CH}$ vibrations. We can then conclude this section by stating that vibrational excitons are most likely absent from the $\Delta v=3$ region of pinenes and chiral cycloalkanones.

\section{Conclusions}

This study provides a calculation model for handling coupled $\mathrm{CH}$-stretching vibrations, whose VCD manifestation is relatively stable and reproducibly repeated in a series of ring compounds [8, 9]; an alternative way of naming the coupled oscillators is to call them vibrational excitons. We notice that Eq. (6), which was devised to describe vibrational excitons, is general for $n$ oscillators and a matrix formalism can be easily and fruitfully constructed starting therefrom; in Section (a) the model is applied to a triplet of states involving two neighbouring $\mathrm{CH}_{2}$ units and a $\mathrm{C}^{*}-\mathrm{H}$ unit at a stereogenic carbon, and its validity stems from being able to correlate the VCD data of several cyclic molecules $[8,9]$, the ring imposing a given conformation to the $\mathrm{CH}_{2} \mathrm{CH}_{2} \mathrm{C}^{*}-\mathrm{H}$ moiety. The DFT/ab initio calculations presented thereafter for three molecules of the original set from Refs. [8, 9] are still in favor of $\mathrm{CH}_{2} / \mathrm{CH}_{2}$ antisymmetric mode coupling, excluding the contribution of $\mathrm{C}^{*}-\mathrm{H}$. To resolve the discrepancy between the results of the two approaches, more cases should be examined experimentally and analyzed by both DFT/ab initio calculations and the vibrational exciton model applied to $n$ modes. We notice that for other chiral molecules, similar conclusions had been reached some time ago [10], mainly on the basis of calculations, even though the considerations of the authors of Ref. [10] might not have sounded as optimistic. We also notice that, in the critically anharmonic region of the $\mathrm{CH}$-stretching fundamentals, some considerations of anharmonic effects should be taken into account; among the latter effects special care should be given to Fermi resonance effects [23], which though should affect VCD bands below $2900 \mathrm{~cm}^{-1}$, where the symmetric $\mathrm{CH}_{2}$-stretching modes are located, and thus make the analysis of the triplet of VCD bands presented above still acceptable. Finally, by assuming that just local modes exist, we obtained an acceptable interpretation of the overtone $\mathrm{CH}$-stretching region at $\Delta v=3$, for the few molecules examined here: we may thus conclude that NIR-VCD spectra below $1300 \mathrm{~nm}$ are dominated by local modes and thus vibrational excitons are either absent or undetectable with the present techniques.

\section{Experimental}

The VCD spectra between 2800 and $3000 \mathrm{~cm}^{-1}$ were taken with the dispersive apparatus described, e.g., in Ref. [9]. They were for ca. $0.05 \mathrm{M}$ solutions in $\mathrm{CCl}_{4}$ in $0.06 \mathrm{~mm}$ pathlength $\mathrm{CaF}_{2}$ cells (see figures captions). The compounds 1, 2, 3, 4 were purchased, mostly from ALDRICH, and not further purified. The deuterated isotopomers $\mathbf{1}-\mathbf{d}_{\mathbf{4}}$ and $\mathbf{2}-\mathbf{d}_{\mathbf{4}}$ were obtained by dissolving the perhydro-parent 
compounds in $\mathrm{D}_{2} \mathrm{O}$ and successive separation, favoring exchange of $\alpha$-hydrogens with deuterium atoms, as described in Refs. [9, 15].

The NIR-VCD spectra were taken by means of the apparatus of Ref. [30], on neat liquid compounds in $5 \mathrm{~mm} \mathrm{SiO}_{2}$ cells (see figures captions), with the methodology described therein.

\section{Acknowledgements}

The first four authors of this paper wish to acknowledge financial support from Italian Ministry of Education, University, and Research through COFIN 2004 fundings.

\section{References}

[1] Davydov AS (1971) Theory of Molecular Exciton. Plenum Press, New York

[2] Frenkel YI (1931) Phys Rev 37: 1276; Frenkel YI (1936) Phys Z Sowjetunion 9: 158

[3] Moffitt WA (1956) J Chem Phys 25: 458

[4] Berova N, Nakanishi K (2000) Exciton Chirality Method: Principles and Applications. In: Berova N, Nakanishi K, Woody RW (eds) Circular Dichroism: Principles and Applications, $2^{\text {nd }}$ ed. Wiley-VCH, New York, p 337

[5] Holzwarth G, Chabay I (1972) J Chem Phys 57: 1632

[6] Keiderling TA, Stephens PJ (1977) J Am Chem Soc 99: 8061

[7] Marcott C, Blackburn CC, Faulkner TR, Moscowitz A, Overend J (1978) J Am Chem Soc 100: 5262

[8] Laux L, Pultz V, Abbate S, Havel HA, Overend J, Moscowitz A, Lightner DA (1982) J Am Chem Soc 104: 4276

[9] Laux L (1982) PhD Thesis. Department of Chemistry, University of Minnesota

[10] Bour P, Keiderling TA (1992) J Am Chem Soc 114: 9100

[11] Schellman JA (1968) Acc Chem Res 1: 144

[12] Devlin FJ, Stephens PJ (1999) J Am Chem Soc 121: 7413

[13] Condon U (1932) Rev Mod Phys 9: 432

[14] Longhi G, Abbate S, Ricard L, Zerbi G (1988) J Chem Phys 92: 3302

[15] Fuhrer H, Kartha VB, Krueger PJ, Mantsch H, Jones RN (1972) Chem Rev 72: 439

[16] Singh RD, Keiderling TA (1981) J Chem Phys 74: 5347

[17] Buffeteau T, Ducasse L, Brizard A, Huc I, Oda R (2004) J Phys Chem A 108: 4080

[18] Stephens PJ (1985) J Phys Chem 89: 748

[19] Stephens PJ (1987) In: Avery J, Dahl JP, Hansen A (eds) Understanding Molecular Properties. Dordrecht, Reidel, p 333

[20] Nafie LA, Freedman TB (2000) In: Berova N, Nakanishi K, Woody RW (eds) Circular Dichroism: Principles and Applications. Wiley-VCH, p 97

[21] Frisch MJ et al. (2003) Gaussian 03, Revision B.05, Gaussian Inc, Pittsburgh, PA

[22] Longhi G, Gangemi R, Lebon F, Castiglioni E, Abbate S, Pultz VM, Lightner DA (2004) J Phys Chem A 108: 5338

[23] Abbate S, Havel HA, Laux L, Pultz V, Moscowitz A (1988) J Phys Chem 92: 3302

[24] Applequist J, Carl JR, Fung KK (1972) J Am Chem Soc 94: 2952; Applequist J (1973) J Chem Phys 58: 4251

[25] Henry BR (1987) Acc Chem Res 20: 429

[26] Child MS, Halonen L (1985) Adv Chem Phys LVII: 1

[27] Abbate S, Gangemi R, Longhi G (2002) J Chem Phys 117: 7575

[28] Marcott C, Faulkner TR, Overend J, Moscowitz A (1977) J Am Chem Soc 99: 8160

[29] Polavarapu P (1996) Mol Phys 85: 1503

[30] Castiglioni E, Lebon F, Longhi G, Abbate S (2002) Enantiomer 7: 161

[31] Nafie LA, Cao X, Guo C (2003) Appl Spectrosc 57: 1245 